\title{
BMJ Open Motivational Interviewing for Maternal Immunisation (MI4MI) study: a protocol for an implementation study of a clinician vaccine communication intervention for prenatal care settings
}

Sarah E. Brewer (1) , ${ }^{1,2}$ Jessica R. Cataldi, ${ }^{3}$ Mary Fisher, ${ }^{1,2}$ Russell E. Glasgow, ${ }^{1,2}$ Kathleen Garrett, ${ }^{4}$ Sean T. O'Leary ${ }^{2,3}$

To cite: Brewer SE, Cataldi JR, Fisher M, et al. Motivational Interviewing for Maternal Immunisation (Ml4MI) study: a protocol for an implementation study of a clinician vaccine communication intervention for prenatal care settings. BMJ Open 2020;10:e040226. doi:10.1136/ bmjopen-2020-040226

- Prepublication history for this paper is available online. To view these files, please visit the journal online (http://dx.doi org/10.1136/bmjopen-2020040226).

Received 07 May 2020 Revised 27 October 2020 Accepted 29 0ctober 2020

Check for updates

(C) Author(s) (or their employer(s)) 2020. Re-use permitted under CC BY-NC. No commercial re-use. See rights and permissions. Published by BMJ.

For numbered affiliations see end of article.

Correspondence to

Dr Sarah E. Brewer;

sarah.brewer@cuanschutz.edu

\section{ABSTRACT}

Introduction Vaccination against influenza and pertussis in pregnancy offers a 'two-for-one' opportunity to protect mother and child. Pregnant patients have increased risk of severe disease from influenza and newborns have increased risk of severe disease from both influenza and pertussis. Obstetricians need communication tools to support their self-efficacy and effectiveness in communicating the importance of immunisation during pregnancy and ultimately improving maternal vaccination rates.

Methods and analysis We describe the protocol for a pragmatic study testing the feasibility and potential impact of a clinician communication strategy on maternal vaccination uptake. This study will be conducted in five prenatal care settings in Colorado, USA. The Motivational Interviewing for Maternal Immunisation strategy involves training prenatal care providers to use motivational interviewing in the vaccine conversation with pregnant patients. Our primary outcomes will be the adoption and implementation of the intervention measured using the Enhanced RE-AIM/Practical Robust Implementation and Sustainability Model for dissemination and implementation. Secondary outcomes will include provider time spent fidelity to Motivational Interviewing and self-efficacy measured through audio recorded visits and provider surveys, patients' visit experience based on audio recorded visits and follow-up interviews, and maternal vaccine uptake as measured through chart reviews.

Ethics and dissemination This study is approved by the following institutional review boards: Colorado Multiple Institutional Review Board. Results will be disseminated through peer-reviewed manuscripts and conference presentations.

Trial registration number NCT04302675.

\section{INTRODUCTION}

Vaccination against influenza and pertussis in pregnancy offers a 'two-for-one' opportunity to protect mother and child. Pregnant patients have increased risk of severe disease from influenza ${ }^{1-4}$ and newborns

\section{Strengths and limitations of this study}

- This study uses a robust dissemination and implementation science framework to assess primary outcomes of the adoption and implementation of a communication strategy for prenatal care providers.

- In measuring vaccination uptake, we will provide preliminary data on the effectiveness of the intervention to improve (1) provider skill and perceived competence and (2) maternal vaccine uptake.

- Qualitative audioelicitation interviews will provide in-depth knowledge about the patient acceptability and necessary adaptations.

- This study will not randomise providers or practices and given the limited sample size cannot provide definitive conclusions regarding effectiveness.

have increased risk of severe disease from both influenza ${ }^{5-7}$ and pertussis. ${ }^{8}{ }^{9}$ The Advisory Committee on Immunisation Practices (ACIP) and American College of Obstetricians and Gynecologists (ACOG) recommend women receive influenza and Tdap vaccine during each pregnancy. ${ }^{10}{ }^{11}$ Despite the benefits of these vaccines and evidence of their effectiveness $^{12-19}$ and safety ${ }^{20-26}$ in pregnancy, uptake of influenza and Tdap vaccination in pregnancy remains low. ${ }^{27-32}$ Influenza vaccination coverage among pregnant women was 54\% for the 2018-2019 season and Tdap coverage was $55 \%,{ }^{33}$ well below the Healthy People 2020 goal of $80 \%{ }^{34}$

Several barriers must be addressed to improve maternal immunisation rates, including patient concerns about the need for and safety of vaccination during pregnancy. ${ }^{35}$ Obstetrics and gynaecology (obgyn) providers report patient concerns including desire for a 'natural pregnancy' and lack of concern about getting influenza. ${ }^{37}$ 
Provider recommendation is associated with receipt of Tdap and influenza vaccines during pregnancy, ${ }^{38}$ and lack of a recommendation is a known barrier. ${ }^{35}$ During the 2018-2019 influenza season, 73\% of pregnant women received a provider recommendation and offer for vaccine $^{33}$ of whom $66 \%$ received the influenza vaccine, showing that a recommendation and offer for vaccination are insufficient to achieve optimal vaccine uptake.

A key issue that this study addresses is the lack of evidence to guide providers in communicating about vaccination during pregnancy. One study of educational text messages showed no impact on maternal influenza vaccination. ${ }^{39}$ Another text messaging study showed a modest impact on influenza vaccine uptake $(49 \%$ vs $47 \%))^{40}$ A multimodal intervention that provided educational materials and evidence-based practice training for clinicians failed to improve maternal immunisation rates. ${ }^{41}$ None of these interventions focused on provider communication about maternal immunisations during clinical encounters. A recent qualitative study of ob-gyn providers revealed a need for provider training in communication techniques to enhance uptake of maternal immunisations. ${ }^{42}$

Little is known about how to address vaccine concerns and communicate about vaccinations with pregnant patients. For childhood vaccines, communication techniques impact vaccine acceptance. ${ }^{43-47}$ For example, presumptive recommendation such as 'Your child needs the measles mumps and rubella (MMR) vaccine today' yields substantially higher acceptance of all vaccines over a participatory recommendation like, 'What do you think about getting the MMR vaccine today? ${ }^{43} 45$ These studies imply that provider education should address vaccine recommendations and how to communicate these recommendations.

Motivational interviewing (MI) is a communication framework that has been shown effective for communicating with vaccine hesitant parents about childhood vaccines. MI is an established, evidence-based collaborative conversational style for strengthening a person's own motivation to change an established, evidence-based, patient-centred framework for behavioural change ${ }^{48-65}$ that is effective even when delivered in a single session. ${ }^{51}{ }^{59}$ MI's core elements-having a person-centred conversation and leveraging inherent motivation for behaviours-make it well suited for use with vaccine hesitant parents given their communication preferences regarding vaccines. ${ }^{667}$ In a large randomised control trial, we found that, paired with a presumptive recommendation, ${ }^{45}$ provider use of MI with parents resulted in increased human papillomavirus vaccine acceptance and improved provider self-efficacy for influencing parental vaccine decisions. ${ }^{68}$ Contrary to some providers initial concerns, providers found MI to be time-efficient, which is crucial as many ob-gyn providers report lacking adequate time to discuss risks and benefits of vaccines with patients. ${ }^{69}$

This manuscript describes the protocol for a pilot implementation study to evaluate the use of MI communication strategies-the Motivational Interviewing for Maternal Immunisation (MI4MI) intervention-on (1) adoption and implementation including audio recorded

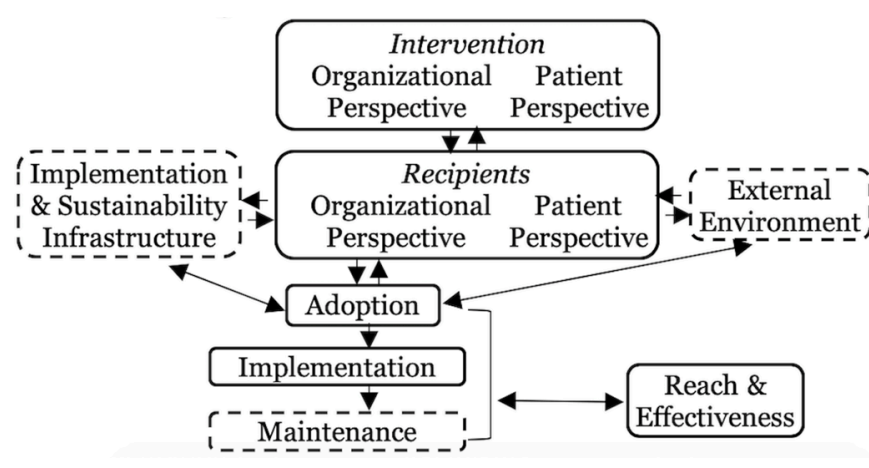

Figure 1 Enhanced Reach Effectiveness Adoption Implementation Maintenance (RE-AIM)/practical robust implementation and sustainability model (PRISM) for evaluation of the MI4MI intervention. MI4MI, Motivational Interviewing for Maternal Immunisation.

clinical encounters; (2) parent and provider experience including self-efficacy and patient autonomy and (3) maternal vaccination status.

\section{Conceptual model}

The application of MI for vaccination in prenatal care settings is based in self-determination theory. ${ }^{70-73}$ This theory holds that motivation to make a positive change (accept a vaccine) is both external and internal, and internal motivation is often related to connecting a decision to one's identity or core values. MI explores ambivalence to behavioural change; is empathetic, informative, non-judgmental and conversational; and involves restating or clarifying a patient's own statements. This process promotes autonomy, builds a sense of competence (self-efficacy), and enhances relatedness between the patient and the provider. It is theorised that these affect internal motivation, thus increasing the likelihood of the positive behaviour change. ${ }^{7072}$ We will assess patient perspectives on self-determination theory concepts of autonomy, competence, and relatedness in qualitative interviews with patients.

The Enhanced Reach Effectiveness Adoption Implementtion Maintenance (RE-AIM)/Practical Robust Implementation and Sustainability Model (PRISM) ${ }^{74} 75$ (see figure 1) of dissemination and implementation (D\&I) will guide development, implementation and assessment of the intervention. PRISM identifies key contextual factors related to the widely used RE-AIM implementation outcomes. We will assess organisational perspective in intervention development and implementation strategies (provider focus groups) and include contextual factors and organisational and patient perspectives in assessing implementation of the intervention (provider focus groups, audiorecorded visit observations and surveys, patient interviews). Future studies will build on this intervention pilot study to address the remaining PRISM elements (sustainability infrastructure and maintenance). For RE-AIM outcomes, we will assess adoption with provider surveys and implementation with 
Table 1 MI4MI trial specifications

\begin{tabular}{|c|c|}
\hline Data category & Information \\
\hline Registry and trial no & ClinicalTrials.gov: NCT04302675 \\
\hline Date of registration & 10 March to 2020 \\
\hline Initial IRB approval date & June 6 to 2019 \\
\hline Title & Adapting MI4MI \\
\hline Countries of recruitment & USA \\
\hline Health condition studied & Maternal vaccination \\
\hline Intervention(s) & clinician vaccine communication strategy \\
\hline Date of first enrolment & $\begin{array}{l}\text { Providers: } 1 \text { May to } 2020 \\
\text { Patients: } 1 \text { September to } 2020\end{array}$ \\
\hline Target sample size & $\begin{array}{l}500 \text { patients with } 2+\text { prenatal care visits for chart reviews } \\
40 \text { audiorecorded visits with audioelicitation interviews, } 20 \text { follow-up interviews }\end{array}$ \\
\hline Trial status & Preintervention \\
\hline Primary outcomes & Adoption and implementation of the MI4MI intervention implementation \\
\hline Key secondary outcomes & $\begin{array}{l}\text { Provider time spent; use of communication techniques; provider self-efficacy in discussing } \\
\text { maternal immunisations with patients; patient experience of vaccine discussions in prenatal } \\
\text { care visits; uptake of maternal vaccines during pregnancy. }\end{array}$ \\
\hline
\end{tabular}

IRB: Institutional Review Board;

MI4MI, Motivational Interviewing for Maternal Immunisation.

audiorecordings and provider reports, and effectiveness with patient interviews and measurement of vaccination rates.

\section{Aim and hypothesis}

The main objective of this study is to evaluate adoption and implementation and pilot test the effectiveness of the MI4MI intervention on maternal immunisation status using a predesign and postdesign. We hypothesise that adoption and implementation of the MI4MI intervention among prenatal care providers will be consistent, feasible and acceptable. We will also test the exploratory hypothesis that the intervention will increase autonomy, competence and connectedness among patients and increase uptake of Tdap and influenza vaccine among pregnant patients.

\section{METHODS}

A summary of the trial's specifications is presented in table 1 .

\section{Study design and registration}

We will conduct a pragmatic pilot study implementing the MI4MI intervention in five Colorado prenatal care practices. This study includes implementation of maternal vaccination communication strategies as well as a preimplementation and postimplementation evaluation of the use of this package of communication strategies. This study is registered with ClinicalTrials.gov (table 1).

\section{Study overview and setting}

The MI4MI intervention will be implemented in five Colorado practices purposively selected to reflect a diversity of practice, provider and patient population characteristics. MI4MI intervention implementation and assessment will involve the same practices and providers who participate in intervention development. Pregnant patients will be recruited from participating practices to assess acceptability of MI among patients. We will time the intervention to occur during influenza season to maximise recruitment. Colorado is the ideal setting for our intervention. Parental vaccine hesitancy is highly prevalent, which is reflected in attitudes towards maternal immunisations. ${ }^{3842}$ Colorado consistently ranks in the top quintile of US states for nonmedical exemptions for childhood vaccination, ${ }^{76}$ and our prior project found that $45 \%$ of pregnant patients surveyed worried about the safety of Tdap and influenza vaccines. $^{38}$ 


\section{Study population and inclusion/exclusion criteria}

All English-speaking and Spanish-speaking patients who receive prenatal care visits at a participating practice during the intervention period of August 2020 to April 2021 will be eligible. All patients included in this study will be pregnant patients. Providers who participate in provider focus groups, surveys and the MI4MI communication training intervention will be men and women. The study settings proposed serve a racially and ethnically diverse population.

Pregnant patients who are less than 18 years of age will be included in the MI4MI intervention because providers may use MI communication techniques in their clinical encounters and no parental permission is required for this portion of the study. Because the interventions include provider communication techniques about recommended vaccinations, the interventions do not fall outside of usual care. Pregnant patients who are less than 18 years of age will also be eligible to participate in audiorecorded encounters and audioelicitation interviews and assent will be obtained. No parental permission is required for this portion of the study.

\section{Recruitment}

\section{Practices}

Practices will be recruited from among prenatal care providers located in Colorado, including urban, suburban and rural communities. Practices will be invited to participate if they have more than two providers (physicians or nurse midwives), are not affiliated with large corporate health systems and stock both influenza and Tdap vaccine routinely. Attempts will be made to recruit a diverse group of five practices, by practice size, setting (urban, suburban and rural), and type of clinicians (ob-gyn physicians, certified nurse midwives, physician assistants and nurse practitioners). The intervention strategies of the MI4MI project will be implemented in participating practices based on their input. These strategies will be performed as part of quality improvement around immunisation delivery within the study practices and are considered evidencebased and the optimal standard of care by the ACIP and Task Force on Community Preventive Services.

\section{Providers}

Providers will be recruited from participating practices for focus groups and provider surveys. All practicing providers will be invited to receive MI4MI trainings and associated maintenance of certification (MOC) and/ or continuing medical education (CME)/continuing nursing education (CNE) credit for participation.

\section{Patients}

We will obtain individual informed consent for audiorecording and audioelicitation patient interviews. Eligible patients will be identified through a brief screening survey assessing vaccine hesitancy, adapted from the paediatric setting, ${ }^{77}$ administered prior to their prenatal care visit. A subset of enrolled patients at participating clinics will have their prenatal care visit with a participating clinician audiotaped to assess clinician-patient vaccine communication practices, including adherence to the MI4MI communication strategy among intervention clinicians (ie, intervention fidelity).

\section{Blinding}

Given our intervention and study design, it is not possible to blind practices or investigators. We will minimise selection bias by approaching all patients who are pregnant and receiving prenatal care at participating practices and by including those who receive prenatal care in the analysis.

\section{Sample size calculations}

This study will implement the MI4MI intervention in five prenatal care practices with between 30 and 45 providers to gather diverse input on adoption and implementation in multiple settings. As a preliminary study, the findings will not be wholly generalisable to all prenatal care settings, but will provide important information for a broader trial.

With 500 charts per sample (100 charts x 5 practices) and an assumed baseline $50 \%$ vaccination rate, we will have $80 \%$ power to detect a $9 \%$ increase in vaccination rates.

We will audiorecord a total of 40 prenatal care visits in which we anticipate maternal vaccination will be recommended. Through audioelicitation interviews with the patients of these visits, we anticipate we will reach thematic saturation with this number of visits.

\section{Intervention}

We will use adult learning theory techniques ${ }^{78}$ that have been found effective in changing provider behaviour ${ }^{79} 80$ to develop a multifaceted MI training programme. ${ }^{81-83}$ These approaches include interactive tailored educational outreach, ${ }^{84}$ rehearsal and coaching, ${ }^{85}$ booster learning sessions $^{85}$ and change agents. ${ }^{86}$ The MI4MI intervention and implementation strategy will include: (1) a video training module introducing presumptive recommendations and the MI4MI communication strategy including rationale and model vaccine discussions; (2) an in-person training session for providers with a brief didactic session followed by provider role-playing and coaching; (3) onepage reference sheets summarising strategies used in MI4MI and examples of key messages; (4) a refresher training sessions 3 months after initial training including question/answer sessions, role-playing and coaching; (5) a study champion identified at each practice to support intervention implementation and liaise with the study team. These components are described in detail in table 2.

\section{Outcomes}

Our primary outcomes will be adoption and implementation of the MI4MI intervention implementation in prenatal care settings. Secondary outcomes include provider time spent, communication technique and 
Table 2 MI4MI intervention components

\begin{tabular}{ll}
\hline Intervention component & Description \\
\hline Online video module & Introduces the MI4MI communication strategy and its rationale
\end{tabular}

One 60 min in-person interactive

clinician training session
(1) a brief didactic session on vaccine hesitancy, how the MI4MI strategy addresses vaccine hesitancy, and practice data on vaccination coverage and vaccine hesitancy prevalence (2) baseline assessments of clinician skills using the presumptive format and $\mathrm{MI}(3)$ modelling the MI4MI intervention followed by clinician rehearsal through role-playing and coaching by the study team

$\begin{array}{ll}\text { Reference sheets } & \begin{array}{l}\text { Provides brief and accessible summaries of the communication behaviours that } \\ \text { comprise MI4MI and example scripted language for key steps in the MI4MI strategy }\end{array} \\ \begin{array}{l}60 \text { min in-person refresher trainings at } 3 \\ \text { months after the start of the intervention }\end{array} & \begin{array}{l}\text { Includes a question and answer session regarding barriers to implementing the } \\ \text { MI4Mlervention followed by role-playing and coaching. Refresher training will } \\ \text { include review of audiotaped encounters to provide feedback for how to improve } \\ \text { incorporation of MI4MI into the vaccine discussion. }\end{array} \\ \text { Practice study champion } & \begin{array}{l}\text { Staff liaison who routinely solicits feedback from intervention clinicians regarding } \\ \text { the Ml4MI intervention and communicates with the study team at regular intervals to } \\ \text { coordinate implementation data collection and assist with implementation issues }\end{array}\end{array}$

MI, motivational interviewing ; MI4MI, Motivational Interviewing for Maternal Immunisation. self-efficacy in discussing maternal immunisations with patients. Exploratory outcomes include the patient experience of vaccine discussions in prenatal care visits and uptake of maternal vaccines.

\section{Data collection methods}

A summary of measures organised by the Enhanced RE-AIM/PRISM dimensions is provided in table 3 .

\section{Adoption and implementation}

To assess MI4MI implementation, we will audiorecord clinical encounters between study providers and pregnant patients. Practice personnel (nurses and medical assistants) will help a study research assistant (RA) identify patients attending prenatal visits. Potential participants will be asked to complete a brief screening questionnaire composed of a validated tool to assess vaccine hesitancy, developed by Oladejo et al, ${ }^{77}$ with additional items about attitudes toward vaccination during pregnancy and influenza vaccine. Hesitant patients will be invited to participate in a study of provider-patient communication at ob-gyn visits in which their clinic visit will be audiorecorded and they will complete 1-2 interviews after the visit. We will describe the study generally to minimise alterations in patient behaviour to meet observer expectations. The RA will obtain written informed consent for recording of the clinic visit. While similar studies have used videorecording, ${ }^{43} 87$ we will use audiorecording without video given the sensitive nature of some elements of the physical exam at prenatal visits. Audiorecorded visits will be analysed for fidelity and adaptation of MI4MI implementation using a structured coding scheme for the key behavioural components of the MI approach in the vaccine discussion. We will recruit throughout the intervention period to assess for waning of intervention implementation over time.
To provide a mixed-methods assessment of adoption and implementation of the MI4MI intervention among providers, we will conduct postintervention focus groups and baseline and postintervention surveys. A postintervention provider focus group with 4-6 providers per practice will be conducted with each practice 9 months after baseline training to assess provider experience using MI, including barriers to applying MI, use of and usefulness of each component of MI4MI training, and how providers adapted the intervention as well as contextual factors. We will conduct postintervention focus groups using the same methods as baseline focus groups.

We will also assess adoption by collecting data on number of providers in each practice, proportion of providers who completed online modules and training session and focus group attendance and reasons for not participating. Surveys will also assess self-reported provider time spent on MI4MI as a preliminary way to investigate intervention cost.

Finally, we will assess organisational contextual factors and practice characteristics using a validated scale for evidence-based vaccination strategies conducted both pre-implementation and at 9 months later with an practice representative. ${ }^{88}$

\section{Provider surveys}

A provider survey will assess provider time and self-efficacy in discussing maternal immunisations with patients. Surveys will be administered by paper or electronically immediately prior to baseline provider training, at 3 months after the initial provider training, and postintervention surveys will be administered at the time of the postintervention provider focus groups approximately 9 months after initial training. Preintervention surveys will collect provider demographic and practice characteristics. The 3-month and 9-month surveys will assess intervention 
Table 3 Outcome measures by RE-AIM/PRISM dimensions

$\begin{array}{llll} & \text { Definition } & \text { Specific measure(S) } & \text { Instrument(S) }\end{array}$

Reach

Proportion of patients with \# of and characteristics of patients with Survey whom trained providers whom provider used Ml versus those not used MI

$\%$ of patient visits in which provider used Ml

\begin{tabular}{ll} 
Adoption & $\begin{array}{l}\text { Proportion and } \\
\text { representativeness } \\
\text { of providers willing to } \\
\text { participate in MI4MI } \\
\text { programme }\end{array}$ \\
\hline Effectiveness & $\begin{array}{l}\text { Average and consistency } \\
\text { of improvement in clinical } \\
\text { outcomes and any } \\
\text { generalisation effects }\end{array}$
\end{tabular}

$\begin{array}{ll}\text { Implementation } & \text { Consistency of delivery } \\ \text { of key intervention } \\ \text { components; adaptations } \\ \text { made to implementation } \\ \text { process; and costs of the } \\ \text { delivery }\end{array}$

Maintenance

Provider and setting intention (in this short term study) to continue or adapt the intervention
Representativeness of those with high versus low levels of implementation

Time spent on $\mathrm{Ml}$ in visits

$\%$ of and characteristics of providers who completed online modules

$\%$ and characteristics of providers who completed training session

$\%$ and characteristics of providers who completed follow-up focus group

$\%$ of pregnant patients receiving

influenza and Tdap vaccines during 2020-2021 influenza season.

Change in patient perceived autonomy, competence and relatedness

Change in provider autonomy and selfefficacy

On all of above, relationship of patient and provider characteristics to these outcomes of

\section{Counts of Ml4MI behavioura} components used in vaccine conversations

Reported changes to MI4MI strategy

End of intervention assessment of intentions: Providers

End of intervention assessment of intentions: Setting

Reasons why or why not
Brief interviews with providers about patients with whome they did not use MI

Administrative records

Brief interview with those who did not participate about reasons

EHR records

Audio-elicitation interviews

Survey

Audiorecorded visits

Focus groups, individual interviews, and review of audio recorded visits

Survey

Survey

Focus Groups

Immunisation Delivery

Scale ${ }^{88}$

Provider focus groups

\begin{tabular}{|c|c|c|c|}
\hline \multicolumn{4}{|l|}{ Other } \\
\hline PRISM contextual factors & $\begin{array}{l}\text { Setting and institutional } \\
\text { factors supporting } \\
\text { or hindering RE-AIM } \\
\text { dimensions }\end{array}$ & $\begin{array}{l}\text { Perceived barriers, facilitators; } \\
\text { procedural incompatibilities, extent to } \\
\text { which other evidence-based practices, } \\
\text { training and resources are already } \\
\text { implemented, practice culture }\end{array}$ & $\begin{array}{l}\text { Immunisation Delivery } \\
\text { Scale } \\
\text { Focus groups }\end{array}$ \\
\hline Patient hesitancy & $\begin{array}{l}\text { The extent to which a } \\
\text { patient is hesitant to } \\
\text { receive maternal vaccines }\end{array}$ & $\begin{array}{l}\% \text { of patients screened with high } \\
\text { hesitancy }\end{array}$ & Screening \\
\hline Pragmatism & $\begin{array}{l}\text { The ability of the MI4MI } \\
\text { strategy to be scaled to } \\
\text { other settings }\end{array}$ & $\begin{array}{l}\text { PRECIS- } 2 \text { score by study team at three } \\
\text { time points }\end{array}$ & PRECIS-2 \\
\hline
\end{tabular}

EHR, electronic health record; MI, motivational interviewing; PRECIS-2, Pragmatic-Explanatory Continuum Indicator Summary-2; PRISM, practical robust implementation and sustainability model; RE-AIM, Reach, Effectiveness, Adoption, Implementation, Maintenance. 
reach by asking providers to estimate the number and proportion of patients with whom they used MI, and reasons for not when they did not. The survey tool will be modified from one used in two prior studies. ${ }^{68} 89$ Completion of both preintervention and postintervention surveys will be required for MOC/CME/CNE credit. The survey will also capture key demographics to be used to understand to what extent provider differences may influence their likelihood to adopt and implement the approach.

\section{Patient interviews}

We will audiorecord a sample of provider visits with vaccinehesitant patients to assess MI4MI implementation (see above). Audioelicitation interviews ${ }^{90}$ will be conducted using the recorded visits to assess the patient perspective of intervention acceptability, perceived autonomy, competence and relatedness. These semistructured audioelicitation interviews will be conducted within 30 days of the index encounter. The study team member will play portions of the recording for the participant to guide the interview. A screening questionnaire will be used to identify and recruit vaccine hesitant patients who may refuse vaccination at their visit, even if MI techniques are used. Participants who do not receive all recommended immunisations at the index visit will be invited to participate in a second interview 3 months later. We will recruit up to 20 participants $(50 \%)$ for follow-up interviews, which will be conducted by phone or in person to assess for change over time in patient perception of maternal immunisations and provider communication.

\section{Vaccine uptake}

To assess preliminary effectiveness of the intervention, we will measure rates of Tdap and influenza vaccination among pregnant patients at study practices using chart review. A study RA will review 100 charts per practice for the preintervention and postintervention influenza seasons (1 October-31 March). Study champions will help the RA identify patients with prenatal visits during the study period and a sample will be selected by a random number generator. Patient demographics, number of clinic visits and Tdap and influenza vaccine eligibility, refusal and receipt will be recorded. ${ }^{91}$

\section{Pragmatic trial design}

Finally, the study team will rate the MI4MI study on its pragmatism for D\&I using the Pragmatic-Explanatory Continuum Indicator Summary-2 instrument at three time points during the project: preimplementation, 3 months after the initial training and at the conclusion of the study. ${ }^{92}$

\section{Participant retention}

Patient participants will receive a US\$50 gift card for participating in an audio-elicitation interview about their audiotaped visit and a second US\$50 gift card for completing a follow-up interview.

Retention of participating clinicians will be supported by the opportunity to earn MOC part IV credit from the
American Board of Obstetrics and Gynaecology and CME or CNE credits. Providers will receive a US $\$ 75$ gift card for participation in postintervention focus groups. Providers who complete preintervention and postintervention surveys and attend all training sessions will receive MOC/ $\mathrm{CME} / \mathrm{CNE}$ credit.

Retention of practices is supported by the study champion. This staff person receives additional support from the study team and compensation to assist with data collection coordination and liaising with the research team.

\section{Data security and storage}

We will create unique study IDs for each patient participant and de-identified data will be stored on a secure and password-protected server. All linkages between the unique study ID and the individual-level data will be destroyed on completion of the study. We will adhere to all Health Insurance Portability and Accountability Act (HIPAA) requirements as required by the law. Data access is limited to study staff, and data are backed up automatically nightly. We will maintain each dataset separately and index records using unique encrypted identifiers to facilitate linkages between files while maintaining confidentiality of personal health information. Analysis of this audiotaped data will be conducted on passwordprotected computers with access restricted to research team members.

\section{Statistical methods}

We will use Pearson's $\chi^{2}$ tests in unadjusted analyses to compare preintervention and postintervention proportions of providers who (1) spent $\geq 5$ min discussing vaccines at typical visits and during visits with pregnant patients who had concerns; (2) used MI techniques in vaccine discussions with pregnant patients (always, frequently, sometimes, never) and (3) perceived they were able to influence patients' vaccine decisions.

Audioelicitation interview and provider focus group data will be analysed using a content analysis approach ${ }^{9394}$ using both deductive and inductive approaches. An a priori codebook will be developed based on the constructs of the self-determination theory and the Enhanced RE-AIM/ PRISM dimensions. Emergent codes will be developed by team consensus.

We will use Pearson's $\chi^{2}$ test in an unadjusted pooled comparison of influenza and Tdap vaccination rates among eligible pregnant patients during preintervention and postintervention periods and logistic regression to adjust for potential covariates, including race, insurance type, number of prenatal care visits and prior vaccination acceptance when available.

\section{Missing data}

We will compare patient and provider characteristics by missing data as well as missing outcome data in all participating practices. We will apply sensitivity analyses techniques to address missing data. 


\section{Patient and public involvement}

Patients involved in this study are pregnant patients seeking prenatal care at participating practices and are only involved in this study as research participants. Patients will not be involved in recruitment, data analysis or dissemination. Clinicians will be involved in the refinement of the MI4MI intervention.

\section{ETHICS AND DISSEMINATION}

All study activities described in this protocol have been approved by the Colorado Multiple IRB. Any protocol modifications will be reviewed and approved by the same.

\section{Informed consent}

Providers who complete the baseline experience survey will be considered to have consented to participation. This survey will contain information about the study and its risks and benefits. For provider focus groups, verbal consent will be performed and a waiver of documentation of consent will be obtained. The only risks to providers are potential loss of confidentiality. Written informed consent will be obtained from patients and clinicians who participate in the audiotaped visits substudy. The study team will record the names of persons participating in the focus group, in order to follow up on themes or issues raised. However, all study results will be published and presented in aggregate form only, with no individual responses identified. The study team will record the names of persons participating in provider surveys in order to track completion for MOC/CME/CNE credit.

Because the entire patient-provider clinical encounter will be audiorecorded, there is potential to capture personal health related information that is unrelated to maternal immunisations and may be considered more private and sensitive in nature. The risks to participants relate to a potential loss of confidentiality. The study team will record the names of persons participating in audiorecording and audioelicitation interviews, in order to facilitate scheduling of interviews after recording.

Individual informed consent will not be required for completion of the chart review portion of the study. This is a retrospective chart review will collect already existing immunisation data recorded as part of routine clinical care.

\section{Monitoring}

The principal investigator of this study (O'Leary) will be responsible for participant safety monitoring. Oversight of study data safety and monitoring will be conducted by a faculty member at UCD who is not involved in the project. This individual will provide independent observation and verification of protocol compliance, recruitment and study progress, and data completeness. The individual will review draft annual reports. They will also monitor the study for adverse events, and the study team's response to these events, should any occur. A letter summarising findings will be included in annual project reports for NIH. Though not anticipated, adverse events will be reported to the IRB promptly, should any occur.

\section{Dissemination plans}

Study materials will be developed so that they may be easily adapted to other prenatal care settings, with particular focus on having the online video module available for use by others immediately. Should this intervention prove effective, we intend to collaborate with other national sites to test the intervention on a broader scale. Results of the study will be presented at national and international research conferences and through peerreviewed publications. Likely of greater impact, we will conduct trainings at key national meetings (eg, ACOG Annual Meeting, Annual Conference on the Science of Dissemination and Implementation in Health, Infectious Diseases Society For Obstetrics And Gynecology Annual Meeting), make our protocol and measures publicly available and seek to have our programme listed on credible sources for Evidence-Based Programmes (eg, ResearchTested Intervention Programs (RTIPS), etc)

\section{Strengths and limitations}

This strength of this study to address the lack of interventions for increasing maternal vaccine uptake include the mixed-methods assessment of adoption and implementation of the MI4MI intervention, a pragmatic model, interventions and measures, the novel implementation of audioelicitation interviews to assess RE-AIM dimensions, and the use of a practice study champion to inform sustainability of implementation in the practice setting.

This study's limitations include a small number of practices and providers in one region of the USA. Practices and providers are not randomised to intervention or control arms and patients are not randomised to receive the MI4MI communication strategy. As such, our sample may not be representative of all practices and those practices that opt to participate may differ from others in immunisation rate or other factors. Additionally, there are limitations to our observation and measurement methods that should be pointed out. First, if providers are aware of being observed they may behave differently than for their other patient visits (Hawthorne effect), which could lead to overestimating adherence to the MI4MI intervention communication strategy in this case. However, evidence suggests that there is little impact on provider or patient behaviour of recording clinical encounters. ${ }^{95-97}$ Second, provider outcomes on survey measures are self-report and at risk for recall and testing biases because they will know the research team is looking for improvement over time and because they will be asked to report on activities over the last 30-60 days without documentation of their activities. Our experience in prior physicians surveys suggests tesing bias is minimal. To reduce recall bias, we will also use audiorecorded visits to assess fidelity to the MI4MI communication strategy 
and validate reports of time spent on vaccination conversations. Third, because pregnancy is a periodic event, we are unable to conduct a longitudinal assessment of women's vaccination uptake across multiple opporutnities for vaccination during pregnancy with and without MI approaches. Fourth, immunisation decisions may differ during influenza season, limiting the generalisability of our findings to this portion of the year. Our prior experience with maternal vaccination suggests this is not the case. However, follow-up evaluation of the maintenance of the MI4MI intervention during the summer months warrants future examination. Finally, the lack of a control group hinders our ability to account for secular trends in vaccination uptake and the impact of other simultaneous efforts to improve vaccination uptake in the practices. Once the MI4MI communication strategy has been adapted to the ob-gyn setting, future studies will be better situated to evaluate the effectiveness of the approach on vaccination uptake. Despite these limitations, this preliminary study will provide important knowledge for scaling an MI intervention for maternal vaccination to other prenatal care settings nationally.

\section{Author affiliations}

${ }^{1}$ Family Medicine, University of Colorado Anschutz Medical Campus, Aurora, Colorado, USA

${ }^{2}$ Adult and Child Consortium for Health Outcomes Research and Delivery Sciences, University of Colorado Anschutz Medical Campus, Aurora, Colorado, USA

${ }^{3}$ Pediatrics, University of Colorado Anschutz Medical Campus, Aurora, Colorado, USA ${ }^{4}$ Colorado School of Public Health, University of Colorado Anschutz Medical Campus, Aurora, CO, United States

Contributors SEB, JRC and STO conceived of the study and intervention and wrote the study protocol. SEB wrote the first draft of the manuscript. JRC, STO, MF, REG and $\mathrm{KG}$ provided input into the study design, intervention development and study protocol, and edited and/or reviewed the manuscript.

Funding This work was supported by the National Institute for Allergies and Infectious Diseases at the US National Institutes of Health, grant number R21Al141822. REG's time supported in part by NCl grant 1P50CA244688-01.

Competing interests None declared.

Patient and public involvement Patients and/or the public were not involved in the design, or conduct, or reporting, or dissemination plans of this research.

Patient consent for publication Not required.

Provenance and peer review Not commissioned; externally peer reviewed.

Open access This is an open access article distributed in accordance with the Creative Commons Attribution Non Commercial (CC BY-NC 4.0) license, which permits others to distribute, remix, adapt, build upon this work non-commercially, and license their derivative works on different terms, provided the original work is properly cited, appropriate credit is given, any changes made indicated, and the use is non-commercial. See: http://creativecommons.org/licenses/by-nc/4.0/.

\section{ORCID iD}

Sarah E. Brewer http://orcid.org/0000-0003-0063-6626

\section{REFERENCES}

1 Creanga AA, Johnson TF, Graitcer SB, et al. Severity of 2009 pandemic influenza A ( $\mathrm{H} 1 \mathrm{~N} 1)$ virus infection in pregnant women. Obstet Gynecol 2010;115:717-26.

2 Hartert TV, Neuzil KM, Shintani AK, et al. Maternal morbidity and perinatal outcomes among pregnant women with respiratory hospitalizations during influenza season. Am J Obstet Gynecol 2003;189:1705-12.

3 Louie JK, Acosta M, Jamieson DJ, et al. Severe 2009 H1N1 influenza in pregnant and postpartum women in California. N Engl J Med 2010;362:27-35

4 Neuzil KM, Reed GW, Mitchel EF, et al. Impact of influenza on acute cardiopulmonary hospitalizations in pregnant women. $\mathrm{Am} \mathrm{J}$ Epidemiol 1998;148:1094-102.

5 Ampofo K, Gesteland PH, Bender J, et al. Epidemiology, complications, and cost of hospitalization in children with laboratoryconfirmed influenza infection. Pediatrics 2006;118:2409-17.

6 Izurieta HS, Thompson WW, Kramarz P, et al. Influenza and the rates of hospitalization for respiratory disease among infants and young children. N Engl J Med 2000;342:232-9.

7 Mullooly JP, Barker WH. Impact of type A influenza on children: a retrospective study. Am J Public Health 1982;72:1008-16.

8 Cortese MM, Baughman AL, Zhang R, et al. Pertussis hospitalizations among infants in the United States, 1993 to 2004. Pediatrics 2008;121:484-92.

9 Vitek CR, Pascual FB, Baughman AL, et al. Increase in deaths from pertussis among young infants in the United States in the 1990s. Pediatr Infect Dis J 2003;22:628-35.

10 Centers for Disease Control and Prevention (CDC). Updated recommendations for use of tetanus toxoid, reduced diphtheria toxoid, and acellular pertussis vaccine (Tdap) in pregnant women-Advisory Committee on Immunization Practices (ACIP), 2012. MMWR Morb Mortal Wkly Rep 2013;62:131-5.

11 Harper SA, Fukuda K, Uyeki TM, et al. Prevention and control of influenza: recommendations of the Advisory Committee on immunization practices (ACIP). MMWR Recomm Rep 2004:53:1-40.

12 Dabrera G, Zhao H, Andrews N, et al. Effectiveness of seasonal influenza vaccination during pregnancy in preventing influenza infection in infants, England, 2013/14. Euro Surveill 2014;19:20959.

13 Eick AA, Uyeki TM, Klimov A, et al. Maternal influenza vaccination and effect on influenza virus infection in young infants. Arch Pediatr Adolesc Med 2011;165:104-11.

14 Zaman K, Roy E, Arifeen SE, et al. Effectiveness of maternal influenza immunization in mothers and infants. $N$ Engl $\mathrm{J} \mathrm{Med}$ 2008;359:1555-64.

15 Poehling KA, Szilagyi PG, Staat MA, et al. Impact of maternal immunization on influenza hospitalizations in infants. Am J Obstet Gynecol 2011;204:S141-8.

16 Benowitz I, Esposito DB, Gracey KD, et al. Influenza vaccine given to pregnant women reduces hospitalization due to influenza in their infants. Clin Infect Dis 2010;51:1355-61.

17 Amirthalingam G, Andrews $\mathrm{N}$, Campbell $\mathrm{H}$, et al. Effectiveness of maternal pertussis vaccination in England: an observational study. Lancet 2014;384:1521-8.

18 Winter K, Cherry JD, Harriman K. Effectiveness of prenatal tetanus, diphtheria, and acellular pertussis vaccination on pertussis severity in infants. Clin Infect Dis 2017;64:9-14.

19 Winter K, Nickell S, Powell M, et al. Effectiveness of prenatal versus postpartum tetanus, diphtheria, and acellular pertussis vaccination in preventing infant pertussis. Clin Infect Dis 2017;64:3-8.

20 Munoz FM, Greisinger AJ, Wehmanen OA, et al. Safety of influenza vaccination during pregnancy. Am J Obstet Gynecol 2005;192:1098-106.

21 Nordin JD, Kharbanda EO, Benitez GV, et al. Maternal safety of trivalent inactivated influenza vaccine in pregnant women. Obstet Gynecol 2013;121:519-25.

22 Kharbanda EO, Vazquez-Benitez G, Lipkind HS, et al. Evaluation of the association of maternal pertussis vaccination with obstetric events and birth outcomes. JAMA 2014;312:1897-904.

23 Berenson AB, Hirth JM, Rahman M, et al. Maternal and infant outcomes among women vaccinated against pertussis during pregnancy. Hum Vaccin Immunother 2016;12:1965-71.

24 Munoz FM, Bond NH, Maccato M, et al. Safety and immunogenicity of tetanus diphtheria and acellular pertussis (Tdap) immunization during pregnancy in mothers and infants: a randomized clinical trial. JAMA 2014;311:1760-9.

25 Shakib JH, Korgenski K, Sheng X, et al. Tetanus, diphtheria, acellular pertussis vaccine during pregnancy: pregnancy and infant health outcomes. J Pediatr 2013;163:1422-6. e1-4.

26 Sukumaran L, McCarthy NL, Kharbanda EO, et al. Association of Tdap vaccination with acute events and adverse birth outcomes among pregnant women with prior Tetanus-Containing immunizations. JAMA 2015;314:1581-7.

27 Ding $\mathrm{H}$, Black CL, Ball S, et al. Influenza vaccination coverage among pregnant women - United States, 2014-15 influenza season. MMWR Morb Mortal Wkly Rep 2015;64:1000-5. 
28 Ding $\mathrm{H}$, Black $\mathrm{CL}$, Ball S, et al. Influenza vaccination coverage among pregnant women--United States, 2013-14 influenza season. MMWR Morb Mortal Wkly Rep 2014;63:816-21.

29 Kharbanda EO, Vazquez-Benitez G, Lipkind HS, et al. Maternal Tdap vaccination: coverage and acute safety outcomes in the vaccine safety Datalink, 2007-2013. Vaccine 2016;34:968-73.

30 Koepke R, Kahn D, Petit AB, et al. Pertussis and influenza vaccination among insured pregnant women - Wisconsin, 20132014. MMWR Morb Mortal Wkly Rep 2015;64:746-50.

31 Ding H, Black CL, Ball S, et al. Influenza Vaccination Coverage Among Pregnant Women - United States, 2016-17 Influenza Season. MMWR Morb Mortal Wkly Rep 2017;66:1016-22.

32 Kerr S, Van Bennekom CM, Liang JL, et al. Tdap Vaccination Coverage During Pregnancy - Selected Sites, United States, 20062015. MMWR Morb Mortal Wkly Rep 2017;66:1105-8.

33 Lindley MC, Kahn KE, Bardenheier BH, et al. Vital Signs: Burden and Prevention of Influenza and Pertussis Among Pregnant Women and Infants - United States. MMWR Morb Mortal Wkly Rep 2019;68:885-92.

34 Van Kalsbeek M, Saunders JB. Healthy people 2020: a road map for health. NCSL Legisbrief 2011;19:1-2.

35 Centers for Disease Control and Prevention (CDC). Influenza vaccination coverage among pregnant women: 2011-12 influenza season, United States. MMWR Morb Mortal Wkly Rep 2012;61:758-63.

36 Schmid P, Rauber D, Betsch C, et al. Barriers of influenza vaccination intention and behavior - a systematic review of influenza vaccine Hesitancy, 2005 - 2016. PLoS One 2017;12:e0170550.

37 O'Leary ST, Riley L, Lindley MC. Vaccine refusal among pregnant women: a national survey of Obstetrician-Gynecologists. IDWeek 2018. San Diego, California, 2017.

38 O'Leary ST, Pyrzanowski J, Brewer SE, et al. Influenza and pertussis vaccination among pregnant women and their infants' close contacts: reported practices and attitudes. Pediatr Infect Dis J 2015;34:1244-9.

39 Moniz MH, Hasley S, Meyn LA, et al. Improving influenza vaccination rates in pregnancy through text messaging: a randomized controlled trial. Obstet Gynecol 2013;121:734-40.

40 Stockwell MS, Westhoff C, Kharbanda EO, et al. Influenza vaccine text message reminders for urban, low-income pregnant women: a randomized controlled trial. Am J Public Health 2014;104:e7-12.

41 Chamberlain AT, Seib K, Ault KA, et al. Improving influenza and Tdap vaccination during pregnancy: a cluster-randomized trial of a multicomponent antenatal vaccine promotion package in late influenza season. Vaccine 2015;33:3571-9.

42 Frew PM, Randall LA, Malik F, et al. Clinician perspectives on strategies to improve patient maternal immunization acceptability in obstetrics and gynecology practice settings. Hum Vaccin Immunother 2018;14:1548-57.

43 Opel DJ, Heritage J, Taylor JA, et al. The architecture of providerparent vaccine discussions at health supervision visits. Pediatrics 2013;132:1037-46.

44 Brewer NT, Hall ME, Malo TL, et al. Announcements versus conversations to improve HPV vaccination coverage: a randomized trial. Pediatrics 2017;139:e20161764.

45 Opel DJ, Mangione-Smith R, Robinson JD, et al. The influence of provider communication behaviors on parental vaccine acceptance and visit experience. Am J Public Health 2015;105:1998-2004

46 Ames HM, Glenton C, Lewin S. Parents' and informal caregivers' views and experiences of communication about routine childhood vaccination: a synthesis of qualitative evidence. Cochrane Database Syst Rev 2017;2:CD011787.

47 Gilkey MB, Calo WA, Moss JL, et al. Provider communication and HPV vaccination: the impact of recommendation quality. Vaccine 2016;34:1187-92.

48 Miller WR, Rollnick S. Motivational interviewing: helping people change. Guilford Press, 2012.

49 Motivational Interview. Available: http://www.motivationalinterview. org/index.html [Accessed 1 Feb 2020].

50 Berg CJ, Thomas JL, An LC, et al. Change in smoking, diet, and walking for exercise in blacks. Health Educ Behav 2012;39:191-7.

51 Brand VS, Bray KK, MacNeill S, et al. Impact of single-session motivational interviewing on clinical outcomes following periodontal maintenance therapy. Int J Dent Hyg 2013;11:134-41.

52 Cooper L. Combined motivational interviewing and cognitivebehavioral therapy with older adult drug and alcohol abusers. Health Soc Work 2012;37:173-9.

53 Leask J, Kinnersley P, Jackson C, et al. Communicating with parents about vaccination: a framework for health professionals. BMC Pediatr 2012;12:154
54 McCain J. To heal the body, get into the patient's head: motivational interviewing: to improve adherence. Biotechnol Healthc 2012;9:10-12.

55 Thomas ML, Elliott JE, Rao SM, et al. A randomized, clinical trial of education or motivational-interviewing-based coaching compared to usual care to improve cancer pain management. Oncol Nurs Forum 2012;39:39-49.

56 Boutin-Foster C, Scott E, Rodriguez A, et al. The trial using motivational interviewing and positive affect and Self-Affirmation in African-Americans with hypertension (triumph): from theory to clinica trial implementation. Contemp Clin Trials 2013;35:8-14.

57 Gabbay RA, Añel-Tiangco RM, Dellasega C, et al. Diabetes nurse case management and motivational interviewing for change (dynamic): results of a 2-year randomized controlled pragmatic trial. $J$ Diabetes 2013:5:349-57.

58 Gance-Cleveland B. Motivational interviewing for adolescent obesity. Am J Nurs 2013;113:11.

59 Hides L, Carroll S, Scott R, et al. Quik fix: a randomized controlled trial of an enhanced brief motivational interviewing intervention for alcohol/cannabis and psychological distress in young people. Psychother Psychosom 2013;82:122-4.

60 Klimas J, Field C-A, Cullen W, et al. Psychosocial interventions to reduce alcohol consumption in concurrent problem alcohol and illicit drug users: cochrane review. Syst Rev 2013;2:3.

61 Nirenberg T, Baird J, Longabaugh R, et al. Motivational counseling reduces future police charges in court referred youth. Accid Anal Prev 2013;53:89-99.

62 Rongkavilit C, Naar-King S, Wang B, et al. Motivational interviewing targeting risk behaviors for youth living with HIV in Thailand. AIDS Behav 2013;17:2063-74.

63 Schoonheim-Klein M, Gresnigt C, van der Velden U. Influence of dental education in motivational interviewing on the efficacy of interventions for smoking cessation. Eur J Dent Educ 2013;17:e28-33.

64 Ski CF, Thompson DR. Motivational interviewing as a brief intervention to improve cardiovascular health. Eur J Cardiovasc Nurs 2013;12:226-9.

65 Tse MMY, Vong SKS, Tang SK. Motivational interviewing and exercise programme for community-dwelling older persons with chronic pain: a randomised controlled study. J Clin Nurs 2013;22:1843-56.

66 Benin AL, Wisler-Scher DJ, Colson E, et al. Qualitative analysis of mothers' decision-making about vaccines for infants: the importance of trust. Pediatrics 2006;117:1532-41.

67 Fredrickson DD, Davis TC, Arnould CL, et al. Childhood immunization refusal: provider and parent perceptions. Fam Med 2004;36:431-9.

68 Dempsey AF, Pyrznawoski J, Lockhart S, et al. Effect of a health care professional communication training intervention on adolescent human papillomavirus vaccination: a cluster randomized clinical trial. JAMA Pediatr 2018;172:e180016.

69 Link-Gelles R, Chamberlain AT, Schulkin J, et al. Missed opportunities: a national survey of obstetricians about attitudes on maternal and infant immunization. Matern Child Health $J$ 2012;16:1743-7

70 Ryan RM, Deci EL. Self-determination theory and the facilitation of intrinsic motivation, social development, and well-being. Am Psychol 2000;55:68-78

71 Ryan RM, Deci EL. Self-determination theory: basic psychological needs in motivation, development, and wellness. Guilford Publications, 2017

72 Ryan RM, Kuhl J, Deci EL. Nature and autonomy: an organizational view of social and neurobiological aspects of self-regulation in behavior and development. Dev Psychopathol 1997;9:701-28.

73 Hagger MS, Protogerou C. Self-determination theory and autonomy support to change healthcare behavior. In: The Wiley Handbook of healthcare treatment engagement: theory, research, and clinical practice, 2020: 141-58.

74 Feldstein AC, Glasgow RE. A practical, robust implementation and sustainability model (PriSM) for integrating research findings into practice. Jt Comm J Qual Patient Saf 2008;34:228-43.

75 Glasgow RE, Harden SM, Gaglio B, et al. RE-AIM planning and evaluation framework: adapting to new science and practice with a 20-year review. Front Public Health 2019;7:64.

76 Seither R, Calhoun K, Mellerson J, et al. Vaccination Coverage Among Children in Kindergarten - United States, 2015-16 School Year. MMWR Morb Mortal Wkly Rep 2016;65:1057-64.

77 Oladejo O, Allen K, Amin A, et al. Comparative analysis of the parent attitudes about childhood vaccines (PACV) short scale and the five categories of vaccine acceptance identified by Gust et al. Vaccine 2016;34:4964-8

78 Merriam SB, Cafrrarella RS. Learing in adulthood. San Francisco, CA Jossey-Bass, 2008. 
79 Bero LA, Grilli R, Grimshaw JM, et al. Closing the gap between research and practice: an overview of systematic reviews of interventions to promote the implementation of research findings. The Cochrane effective practice and organization of care review group. BMJ 1998;317:465-8.

80 Grimshaw JM, Eccles MP, Walker AE, et al. Changing physicians' behavior: what works and thoughts on getting more things to work $J$ Contin Educ Health Prof 2002;22:237-43.

81 Davis DA, Thomson MA, Oxman AD, et al. Changing physician performance. A systematic review of the effect of continuing medical education strategies. JAMA 1995;274:700-5.

82 Grimshaw JM, Shirran L, Thomas R, et al. Changing provider behavior: an overview of systematic reviews of interventions. Med Care 2001;39:II2-45.

83 Bauchner H, Simpson L, Chessare J. Changing physician behaviour. Arch Dis Child 2001;84:459-62.

84 O'Brien MA, Rogers S, Jamtvedt G, et al. Educational outreach visits: effects on professional practice and health care outcomes. Cochrane Database Syst Rev 2007;308:CD000409.

85 Rao JK, Anderson LA, Inui TS, et al. Communication interventions make a difference in conversations between physicians and patients: a systematic review of the evidence. Med Care 2007;45:340-9.

86 Dearing JW. Applying diffusion of innovation theory to intervention development. Res Soc Work Pract 2009;19:503-18.

87 Waitzkin $\mathrm{H}$. On studying the discourse of medical encounters. A critique of quantitative and qualitative methods and a proposal for reasonable compromise. Med Care 1990;28:473-88.

88 O'Leary ST, Pyrzanowski J, Brewer SE, et al. Evidence-based vaccination strategies in obstetrics and gynecology settings: current practices and methods for assessment. Hum Vaccin Immunother 2016;12:866-71.

89 Chamberlain AT, Limaye RJ, O'Leary ST, et al. Development and acceptability of a video-based vaccine promotion tutorial for obstetric care providers. Vaccine 2019;37:2532-6.

90 Henry SG, Fetters MD. Video elicitation interviews: a qualitative research method for investigating physician-patient interactions. Ann Fam Med 2012;10:118-25

91 Brewer SE, Barnard J, Pyrzanowski J, et al. Use of electronic health records to improve maternal vaccination. Womens Health Issues 2019;29:341-8.

92 Lipman PD, Loudon K, Dluzak L, et al. Framing the conversation: use of PRECIS-2 ratings to advance understanding of pragmatic trial design domains. Trials 2017;18:532.

93 Elo S, Kyngäs $\mathrm{H}$. The qualitative content analysis process. J Adv Nurs 2008;62:107-15.

94 Hsieh H-F, Shannon SE. Three approaches to qualitative content analysis. Qual Health Res 2005;15:1277-88.

95 Pringle M, Stewart-Evans C. Does awareness of being video recorded affect doctors' consultation behaviour? Br J Gen Pract 1990;40:455-8.

96 Henry SG, White AEC, Magnan EM, et al. Making the most of video recorded clinical encounters: optimizing impact and productivity through interdisciplinary teamwork. Patient Educ Couns 2020;103:2178-84.

97 Themessl-Huber M, Humphris G, Dowell J, et al. Audio-visual recording of patient-GP consultations for research purposes: a literature review on recruiting rates and strategies. Patient Educ Couns 2008;71:157-68. 